\title{
Identification of somatic mutations using whole-exome sequencing in Korean patients with acute myeloid leukemia
}

Seong Gu Heo ${ }^{1,2}$, Youngil Koh ${ }^{3}$, Jong Kwang Kim ${ }^{4,5}$, Jongsun Jung ${ }^{6}$, Hyung-Lae Kim, Sung-Soo Yoon ${ }^{3}$ and Ji Wan Park ${ }^{1 *}$ (i)

\begin{abstract}
Background: Acute myeloid leukemia (AML) is a biologically and clinically heterogeneous cancer of the bone marrow that is characterized by the rapid growth of abnormal myeloid cells.

Methods: We performed a mutational analysis to identify AML somatic mutations using the whole-exome sequencing data of 36 tumor-normal sample pairs from Korean patients with de novo AML. We explored the functional impact of the genes identified in the mutational analyses through an integrated Gene Ontology (GO) and pathway analysis.

Results: A total of 11 genes, including NEFH $\left(p=6.27 \times 10^{-13}\right.$ and $\left.q=1.18 \times 10^{-8}\right)$ and TMPRSS13 $\left(p=1.40 \times 10^{-10}\right.$ and $q=1.32 \times 10^{-6}$ ), also demonstrated $q$ values less than 0.1 in 36 Korean AML patients. Five out of the 11 novel genes have previously been reported to be associated with other cancers. Two gene mutations, CEBPA ( $p=5.22 \times$ $10^{-5}$ ) and $\operatorname{ATXN3}\left(p=9.75 \times 10^{-4}\right)$, showed statistical significance exclusively in the M2 and M3 subtypes of the French-American-British classifications, respectively. A total of 501 genes harbored 478 missense, 22 nonsense, 93 frameshift indels, and/or three stop codon deletions and these gene mutations significantly enriched GO terms for signal transduction (GO:0007165, $p=1.77 \times 10^{-3}$ ), plasma membrane (GO:0005886, $\left.p=3.07 \times 10^{-4}\right)$, and scaffold protein binding (GO:0097110, $\left.p=8.65 \times 10^{-4}\right)$. The mitogen-activated protein kinase (hsa04010, $\left.7.67 \times 10^{-4}\right)$ was the most enriched Kyoto Encyclopedia of Genes and Genomes pathway.

Conclusions: Morphological AML subtypes may in part reflect subtype specific patterns of genomic alterations. Following validation, future studies to evaluate the usefulness of these genes in genetic testing for the early diagnosis and prognostic prediction of AML patients would be worthwhile.
\end{abstract}

Keywords: Acute myeloid leukemia, Gene ontology, Pathway analysis, Somatic mutation, Subtype-specific mutation, Whole-exome sequencing

\section{Background}

Acute myeloid leukemia (AML) is a highly malignant cancer of the bone marrow that is characterized by the rapid growth of abnormal myeloid cells. In 2012, leukemia accounted for $2.5 \%$ and $3.2 \%$ of all new cancer cases and deaths worldwide, respectively [1]. The incidence rate of leukemia increased from 4.7 to 5 cases per 10,000 Koreans from 1999-2010 [2]. AML cytogenetic

\footnotetext{
* Correspondence: jwpark@hallym.ac.kr

${ }^{1}$ Department of Medical Genetics, College of Medicine, Hallym University, 1 Hallymdaehak-gil, Chuncheon, Gangwon-do 24252, Republic of Korea Full list of author information is available at the end of the article
}

studies provide important diagnostic and prognostic information for AML patients. However, approximately $50 \%$ of AML patients have a normal karyotype (NKAML). Although Schlenck et al. showed that the combination of the mutations in FLT and NPM1 or CCAAT/ enhancer binding protein (C/EBP), alpha (CEBPA) could be used to predict NK-AML prognosis, most patients did not have this mutation set [3]. This finding suggests that AML is a highly heterogeneous disease and that a large number of causal mutations have not yet been uncovered [4]. Two systems, the French-American-British (FAB) classification and the newer World Health 
Organization (WHO) classification, have been used to classify AML into subtypes. To compare our results with the previous studies, we used the FAB system, the most commonly used classification in previous sequencing studies for AML, including The Cancer Genome Atlas (TCGA) whole genome sequencing (WGS) [5, 6]. AML is classified into eight subtypes (M0 through M7) according to the FAB classification based on its morphological features: early forms in white blood cells (M0-M5), red blood cells (M6), and platelets (M7) [3].

Next-generation sequencing technologies have extended AML genetic studies to a genome-wide scope at a singlebase resolution. Ley et al. (2008) performed the first AML WGS study on one Caucasian woman with the cytogenetically normal AML subtype M1 and reported nonsynonymous single nucleotide variants (nsSNVs) in eight genes (i.e., CDH24, PCLKC, GPR123, EBI2, PTPRT, $K N D C 1, S L C 15 A 1$, and GRINL1B) and insertions in the coding regions of the FLT3 and NPM1 genes [7]. Mardis and his colleagues reported that $16 \%$ of 80 NK-AML patients had a somatic point mutation in the $I D H 1$ gene [8]. According to a later report by Ley et al. (2010), approximately $22 \%$ of 281 AML patients had DNMT3A mutations that were newly discovered using targeted sequencing [9]. In a Chinese study using targeted exome sequencing, the patients with the DNMT3A Arg882 mutation showed poor prognosis among AML-M5 individuals [10]. A WGS study with eight Caucasian AML patients showed clonal evolution patterns and mutations associated with relapsed AML in the genes $W A C, S M C 3, D I S 3$, $D D X 41$, and $D A X X$ [11]. Recently, TCGA analyzed 50 and 150 patients with de novo AML using WGS and wholeexome sequencing (WES), respectively. They found 23 significantly mutated genes and 237 gene mutations that recurred in at least two patients, which were grouped into nine categories according to their biological functions [6].

AML is a clinically and genetically heterogeneous disease, hence discovering subtype-specific mutations may provide additional prognostic information for AML patients. In this study, we aimed to replicate previous findings in the European studies and to characterize the landscape of somatic mutations present in Korean acute myeloid leukemia. We also performed a stratified analysis for FAB M2- and M3-subtypes to investigate if certain mutations have subtype-specific effects. We subsequently evaluated the functional properties of the significantly mutated genes using an integrated systems analysis of Gene Ontology (GO) and biological pathways.

\section{Methods}

\section{Patients and samples}

We included 36 Korean patients with de novo AML who visited the Division of Hematology and Medical Oncology, Seoul National University Hospital, from 1995 to 2013 who had not received a bone marrow transplant prior to sampling. All subjects provided matched tumor-normal sample pairs that passed a DNA quality control (QC) test. The Institutional Review Board for Human Research at Seoul National University approved the study protocol (IRB number 1201-099-396), and all participants signed informed consent forms for WES. A clinician conducted a retrospective medical record review to obtain clinical data including disease status and blood chemistry. An approximately $10-\mathrm{mL}$ bone marrow sample was aspirated from each participant by a clinician in an aseptic environment, and genomic DNA was isolated using the QIAamp DNA Blood Maxi Kit following the manufacturer's instructions (Qiagen, Inc., Valencia, CA, USA). A 2-ml whole-saliva sample was obtained from the same individuals for the matched normal samples, and the genomic DNA was extracted using the Oragen DNA Self-Collection kit (DNA Genotek, Inc., Ontario, Canada).

\section{Whole-exome sequencing}

We captured the target DNA sample using the Agilent SureSelect Human All Exon $50 \mathrm{Mb}$ Kit (Agilent Technologies Inc., Santa Clara, CA, USA). The entire exome regions for both the tumor and normal samples from 36 AML patients were sequenced using the HiSeq 2000 platform with a 100 bp paired-end read protocol (Illumina, Inc. San Diego, CA, USA). Each the tumor and the normal sample were sequenced to an average read depth of 76X. The fastq quality score and read length cutoff determined by the NGS QC-toolkit were set to 20 and 70 , respectively [12]. We aligned the filtered reads to the reference assembly of human_g1k_v37 fasta using BWA-0.7.5 [13] and called somatic SNVs using MuTect v1.1.4 according to the Catalogue of Somatic Mutations in Cancer (COSMIC) v68 database [14]. We used Varscan v2.3.6 to call short indels [15]. We obtained translational effect of variant allele, protein change information, mutation reports in Catalog of Somatic Mutations in Cancer, and predictions of coding non-synonymous variants on protein function using the Oncotator web application [16]. Preliminary reports using six of 36 samples have been previously published elsewhere (Additional file 1: Table S1) [17-19].

\section{Statistical analysis}

We performed a mutation significance test using MutSigCV v1.4 with a significance threshold of a $p$ value less than 0.05 . The $p$ value was calculated with the chi-square test to determine whether the observed mutations in a gene significantly exceeded the expected frequency of random background mutations. We additionally considered a $q$ value less than 0.1 , which was an analogue of the $p$ value calculated based on the Benjamini-Hochberg false discovery rate (FDR) [20]. To 
identify subtype-specific mutant genes, we performed the test individually in each of the subgroups as follows: M2AML, acute myeloblastic leukemia with maturation, and M3-AML, acute promyelocytic leukemia. We screened for mutations that recurred in more than two patients with a $p$ value less than 0.05 in any patient group. We categorized a mutant gene as a subtype-specific gene if the statistical significance of any one subgroup (i.e., M2 or M3) represented exclusively the significance of the total patient group. Finally, we systematically searched the PubMed database (www.ncbi.nlm.nih.gov/pubmed) to review previous studies on the relevance of the genes with $p$ values less than 0.05 and $q$ values less than 0.1 for AML and/or other cancers.

\section{Gene set enrichment analysis and pathway analysis}

To investigate the biological relevance of the mutations, we performed a GO enrichment analysis with the Database for Annotation, Visualization, and Integrated Discovery (DAVID 6.8 beta) [21]. We categorized the function of these genes into three classes: 'biological process', 'cellular components', and 'molecular function'. We also used the Kyoto Encyclopedia of Genes and Genomes (KEGG) database (http://www.genome.jp/ kegg/pathway.html) to identify pathways for the genes that were frequently mutated in AML cells [22].

\section{Results}

The mean age of the 36 AML patients was approximately 46 years. The mean percentage of blasts in the bone marrow $(60.93 \%)$ in patients in this study was approximately 12 -fold higher than the level in patients undergoing complete remission. Overall, the abnormally high WBC count and low platelet count observed in the study subjects represent typical characteristics of AML patients. A total of 32 patients were grouped into five AML subtypes as follows: M1 $(n=43)$, M2 $(n=11)$, M3 $(n=12)$, M4 $(n=3)$, and M5 $(n=3)$. However, four patients were not placed into any of the AML subgroups (Table 1). Details of the 36 AML patients including sex, age, FAB subtype, and cytogenetic abnormalities were shown in Additional file 1: Table S1. Ten of 11 patients with the acute promyelocytic leukemia (APL)-specific chromosomal translocations $\mathrm{t}(15 ; 17)$ (q22; $\mathrm{q} 21)$ were classified into the FAB subtype M3. Eight of 12 AML patients with normal karyotype fell into the M2 subtype.

\section{Mutation detection}

We identified a total of 4,954 intragenic somatic mutations and 43 genes passed a mutation significance $p$ value threshold 0.01 (data not shown). Among them, 11 genes demonstrated $q$ values less than 0.1 in 36 AML patients (Table 2). The most significantly mutated gene in AML cells was the heavy polypeptide gene (NEFH, $p=6.27 \times$
Table 1 Characteristics of the 36 acute myeloid leukemia patients

\begin{tabular}{ll}
\hline Variables & AML $(n=36)$ \\
\hline Age, mean (SE) & $46.43(3.09)$ \\
Male (\%) & $22(61.11)$ \\
Absolute neutrophil count $\times 10^{3} / \mathrm{mm}^{3}$, mean (SE) & $1.99(0.92)$ \\
Bone marrow blast (\%), mean (SE) & $60.93(4.59)$ \\
White blood cell count $\times 10^{3} / \mathrm{mm}^{3}$, Mean (SE) & $25.72(4.99)$ \\
Platelet count $\times 10^{3} / \mathrm{mm}^{3}$, Mean (SE) & $73.34(13.03)$ \\
Overall survival time (months), mean (SE) & $76.84(31.08)$ \\
French-American-British ${ }^{\mathrm{a}}, \mathrm{n}$ & \\
M1 & 3 \\
M2 & 11 \\
M3 & 12 \\
M4 & 3 \\
M5 & 3 \\
Others ${ }^{b}$ & 4 \\
\hline
\end{tabular}

Abbreviation: $A M L$ acute myeloid leukemia, $S E$ standard error

${ }^{\mathrm{a}} \mathrm{AML}$ is classified into five subtypes (M1 through M5) according to the FrenchAmerican-British (FAB) classification

${ }^{\mathrm{b}}$ Others are comprised of myelodysplastic syndrome $(n=3)$ or whose subtype information was not available $(n=1)$

$\left.10^{-13}, q=1.18 \times 10^{-8}\right)$. Frameshift indels potentially lead to the disease since they alter protein amino acid sequences which were found in olfactory receptor family 2 subfamily $\mathrm{T}$ member 35 (OR2T35) and proprotein convertase subtilisin/kexin type 5 (PCSK5). Missense SNVs were found in transmembrane protease, serine 13 gene (TMPRSS13) in six samples, keratin associated protein 45 (KRTAP4-5) in one sample, and G protein regulated inducer of neurite outgrowth 1(GPRIN1) in one sample (Table 2 and Additional file 1: Table S2).

\section{Subtype-specific somatic mutations}

We identified three significantly mutated genes with a $p$ value of less than 0.01 for each M2 and M3 group. Among them, we identified two genes, CEBPA (19q13.1, $\left.p=5.22 \times 10^{-5}\right)$ and E1A binding protein $\mathrm{p} 400$ (EP400, $\left.12 \mathrm{q} 24.33, p=4.45 \times 10^{-4}\right)$, that showed statistical significance exclusively in the M2 subtype, as well as one gene, ataxin 3 (ATXN3, $\left.p=9.75 \times 10^{-4}\right)$ that was exclusive for the M3 subtype. In other words, these mutations were found to be more frequent in either M2 or M3 subgroup cases than in the total cohort of 36 AML patients (Table 2). Although only eight genes identified in all cases after Bonferroni correction for multiple testing (with a $p$ value cutoff of $1 \times 10^{-5}$ for the 4,954 genes under test), 10 among the 16 genes listed in Table 2 were cancer-relevant genes reported by previous studies. Especially, early studies revealed an association between the CEBPA mutations and AML. Figure 1 summarizes the results of our mutational analysis. The $-\log 10$ of the $p$ value for each mutation (y-axis) was plotted against 
Table 2 Genes identified through mutational analysis in AML in the total, M2, and M3 subtype patient groups

\begin{tabular}{|c|c|c|c|c|c|}
\hline Gene & Chr & Type of variant (patients, n) & $p^{\mathrm{a}}$ & $q^{\mathrm{a}}$ & Previous studies $^{\mathrm{b}}$ \\
\hline \multicolumn{6}{|c|}{$\overline{A M L}(p<0.01$ and $q<0.10)$} \\
\hline NEFH & 22 & In Frame Ins(6)/Del(4) & $6.27 \times 10^{-13 c}$ & $1.18 \times 10^{-8}$ & RCC [23], ESCC [24] \\
\hline TMPRSS13 & 11 & In Frame Del(6), Missense(5) & $1.40 \times 10^{-10 c}$ & $1.32 \times 10^{-6}$ & \\
\hline KRTAP4-5 & 17 & In Frame Ins(5), Missense(1) & $5.07 \times 10^{-8 c}$ & $3.19 \times 10^{-4}$ & \\
\hline OR2T35 & 1 & Frame Shift Ins(1)/Del(4) & $1.50 \times 10^{-7 c}$ & $7.09 \times 10^{-4}$ & \\
\hline HAVCR1 & 5 & Intron SNV(1), In Frame Del(5), Silent(1) & $3.62 \times 10^{-7 c}$ & $1.37 \times 10^{-3}$ & Colorectal [25], RCC [26] \\
\hline IFI27 & 14 & In Frame Ins(3) & $6.88 \times 10^{-6 c}$ & 0.02 & Ovarian [27], Breast [28] \\
\hline PCSK5 & 9 & Intron SNV(1), Frame Shift Del(6), 3'UTR Ins(7)/Del(1) & $8.50 \times 10^{-6 c}$ & 0.02 & Lung [29] \\
\hline GPRIN1 & 5 & Missense(1), In Frame Del(5) & $8.88 \times 10^{-6 c}$ & 0.02 & \\
\hline MRPL18 & 6 & In Frame Del(3) & $2.09 \times 10^{-5}$ & 0.04 & \\
\hline ARSD & $x$ & Intron Ins(2)/Del(2), In Frame Del(4) & $2.93 \times 10^{-5}$ & 0.05 & CLL [30] \\
\hline MAML3 & 4 & In Frame Ins(2)/Del(4), Silent(3), Intron Del(1) & $3.33 \times 10^{-5}$ & 0.05 & \\
\hline \multicolumn{6}{|c|}{ AML-M2 $(p<0.01)$} \\
\hline CEBPA & 19 & In Frame Ins(3) & $5.22 \times 10^{-5}$ & 0.49 & HCC [31], AML [32] \\
\hline EP400 & 12 & Intron Del(1), In Frame Ins(4)/Del(1) & $3.45 \times 10^{-4}$ & 1 & RCC [33], Colorectal [34] \\
\hline \multicolumn{6}{|c|}{ AML-M3 $(p<0.01)$} \\
\hline ATXN3 & 14 & In Frame Ins(2), Intron Ins(1) & $9.75 \times 10^{-4}$ & 1 & Lung [35] \\
\hline
\end{tabular}

Abbreviation: AML acute myeloid leukemia, B-CLL B-cell chronic lymphocytic leukemia, Breast breast cancer, Colorectal colorectal cancer, CLL chronic lymphocytic leukemia, Chr chromosome, Del deletion, ESCC esophageal squamous cell carcinoma, HCC hepatocellular carcinoma, Ins insertion, Lung lung cancer, MDS myelodysplastic syndrome, NMSC non-melanoma skin cancer, Ovarian ovarian cancer, RCC renal cell carcinoma, SNV single nucleotide variant

${ }^{a} p$ and $q$ values were obtained from mutational analysis

${ }^{b}$ Cancers reported by previous studies to have associations with the gene mutations

'The genes still being significant after Bonferroni correction

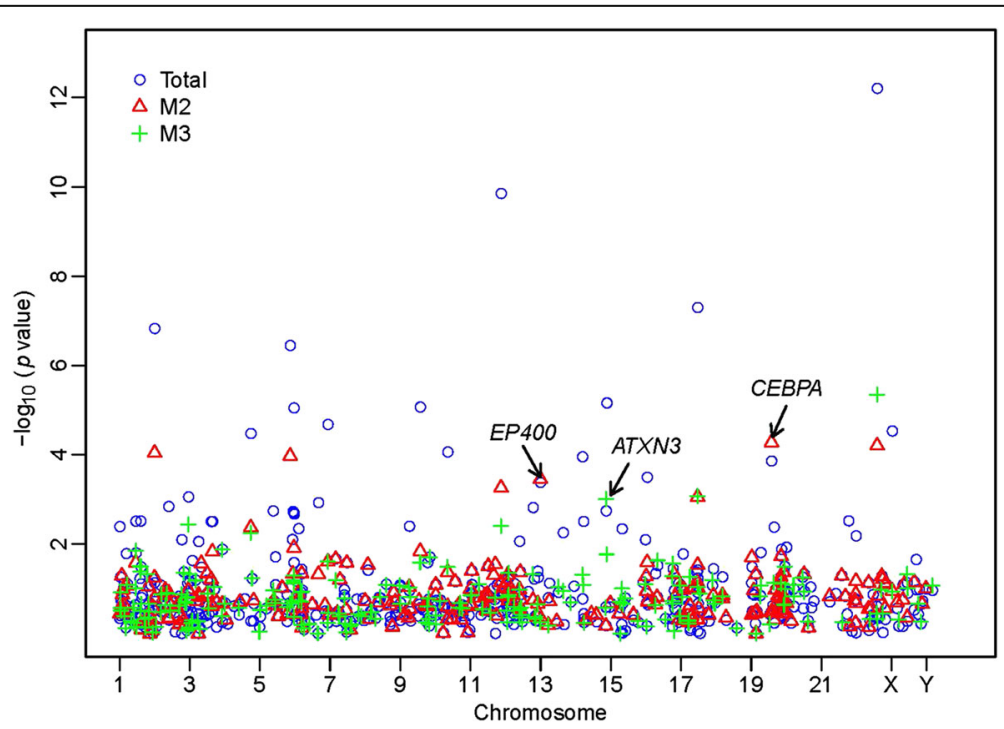

Fig. 1 Three genes demonstrating AML subtype specificity $(p<0.01)$. The symbols, circle $(0)$, triangle $(\Delta)$, and cross $(+)$ denote the significance levels of each gene estimated in the total, M2- and M3-subtype groups, respectively. The labeled genes demonstrate that their mutational significance levels are higher in a subtype group than in the total group 
their respective chromosomal position ( $\mathrm{x}$-axis). Specifically, the three subtype-specific mutant genes with a $p$ value of less than 0.01 were labelled in the plot.

\section{Pathways and biological processes}

We identified total of 501 genes harbored 478 missense, 22 nonsense, 93 frameshift indels, and/or three stop codon deletions. We performed GO and KEGG pathway enrichment analyses with the input consisted of the 501 genes. The GO analysis demonstrated that 43 mutated genes could contribute to AML tumorigenesis through the biological processes such as signal transduction $(p=$ $1.77 \times 10^{-3}$, Fold Enrichment $=1.63$ ). In terms of cellular components, the plasma membrane showed the most significant alteration $\left(p=3.07 \times 10^{-4}\right.$, Fold Enrichment $=$ 1.32). Scaffold protein binding was the most significantly altered molecular function $\left(p=8.65 \times 10^{-4}\right.$, Fold Enrichment $=6.18$ ). To investigate the pathway-level relationship of the mutated genes, we performed a pathway analysis in the KEGG database (Additional file 1: Table S3). Mitogen-activated protein kinase signaling $\left(p=7.67 \times 10^{-4}\right.$, Fold Enrichment $=2.50)$ and long-term potentiation $(p=$ $2.40 \times 10^{-3}$, Fold Enrichment $=4.28$ ) pathways were significantly altered in AML.

\section{Discussion}

A total of 11 genes were significantly mutated in 36 AML patients $(p<0.01$ and $q<0.1)$. Specifically, NEFH, hepatitis A virus cellular receptor 1 (HAVCR1, 5q33.2), interferon, alpha-inducible protein 27 (IFI27, 14q32), PCSK5, and the arylsulfatase D gene (ARSD, Xp22.3) have been implicated in a variety of cancers. For instance, the variants of $N E F H$ gene, a tumor suppressor, were suggested as prognostic markers for renal cell carcinoma (RCC) and contributed to susceptibility of esophageal squamous cell and hepatocellular carcinomas $[24,36]$. While overexpression of this gene interrupts the development of cell structure and function in normal cells [37], loss-of-function mutations in this gene activate the Akt/ $\beta$-catenin pathway and cause increased glycolysis and result in mitochondrial dysfunction in cancer cells [24]. The transmembrane protease, serine 13 gene (TMPRSS13, 11q23), a splice variant of mosaic serine protease large form (MSPL), encodes a family of the type II transmembrane serine proteases which plays critical roles in maintaining homeostasis, infection, and tumorigenesis $[38,39]$. The HAVCR1 gene is a biomarker for diagnosing renal cell, ovarian, and colorectal carcinoma [25, 40]. Especially, elevated expression of this gene prevents cancer cell invasion and adhesion in colorectal cancer cells [25]. IFI27, the most highly upregulated gene in human whole blood, is related with immune response through activation of $\mathrm{T}$ lymphocytes and dendritic cells [41]. Furthermore, this gene induces the interferon-alpha and stimulates myeloid dendritic cells [42]. The proprotein convertases (PCs) play important roles in development and metastasis of multiple cancers. The PCSK5 gene (also known as PC5 or PC6) has been reported to be systematically downregulated in intestinal tumors of the knockout mouse model and human [43].

The protein encoded by the ARSD gene located on $\mathrm{X}$ chromosome is a member of the sulfatase family, which is an essential element for skeletal and cartilage growth. The elevated expression of this protein was suggested to be associated with lipid metabolism such as sphingolipid and development of chronic lymphocytic leukemia [30]. A cluster of Mastermind-like (MAML) genes, including $M A M L 1, M A M L 2$, and $M A M L 3$, encodes transcriptional co-activators for various signal pathways such as Notch signaling and tumor suppressor pathway activated in multiple cancers [44]. Specifically, the MAML3 gene regulates the retinoic acid gene, which inhibits growth of human tumor cells [45]. We identified four novel genes, KRTAP4-5, OR2T35, GPRIN1, and MRPL18, of unknown function in tumor progression and metastasis.

The genes mutated exclusively in the patients with the AML subtypes M2 and M3 have been reported more frequently in previous studies to have an association with AML and/or other types of cancers than the genes identified in the total AML patient group. For instance, previous NGS studies for AML evaluated the prognostic impact of the gene CEBPA in AML patients $[6,32]$. This gene had the lowest $p$ value in the AML M2 group in the current study. The high proportion of M2-patients in the study subjects may have affected the results of previous studies. The E1A binding protein p400 (EP400, 12q24.33) was reported to be associated with cancers such as RCC and colorectal cancer. All three genes specific to the M3 subtype (ATXN3, thymine-DNA glycosylase (TDG, 12q24.1), and HCLS) also showed possible associations with cancer risks, such as B-cell chronic lymphocytic leukemia (B-CLL).

Gene set enrichment analysis showed that MAPK signaling pathway was significantly enriched in the 36 Korean AMLs. MAPKs play important role in converting extracellular stimuli into cellular responses and are often altered in cancers [46]. The genomic region, Chromosome 11:117789342-117789345, harbor five missense mutations leading to protein changes, p.A77G and p.Q78R, of transmembrane serine proteases that is known to function in tumorigenesis [39]. We additionally investigated the clinical features of patients who share the same recurrent mutations, including age, absolute neutrophil count, bone marrow blast percentage, white blood cell count, platelet count, and overall survival time, however there were no specific clinical features observed among them. 


\section{Conclusions}

In this study, we replicated multiple gene mutations reported by previous European studies in Korean patients. We also discovered novel genes significantly mutated in AMLs and some mutated genes that showed subtype-specific patterns of mutations. The effects of novel genes and subtype-specific somatic mutations in AML warrant further validation in larger cohorts. Following validation, it would be worthwhile in future studies to evaluate the usefulness of these genes in genetic testing for the early diagnosis and prediction of the prognosis of AML patients.

\section{Additional file}

Additional file 1: Table S1. Details of the $36 \mathrm{AML}$ patients. Table S2.

Functional information for 15 significantly mutated genes in 36 Korean AML patients. Table S3. Results of gene ontology and KEGG pathway analyses. (DOCX $38 \mathrm{~kb})$

\section{Abbreviations}

AML: Acute myeloid leukemia; ANK-AML: AML with an abnormal karyotype; APL: Acute promyelocytic leukemia; $\mathrm{CPH}$ : Cox proportional hazards regression; FAB: French-American-British classification; FDR: False discovery rate; GO: Gene ontology; IGV: Integrative genomics viewer; INO80: INO80 complex subunit; JMML: Juvenile myelomonocytic leukemia; LR: Logistic regression; MAPK: Mitogen-activated protein kinase; NGS: Next-generation sequencing technologies; NK-AML: AML with a normal karyotype; nsSNVs: Non-synonymous single nucleotide variants; OR: Odds ratio; TCGA: The Cancer Genome Atlas Research Network; WBC: White blood cells; WES: Whole-exome sequencing; WGS: Whole genome sequencing

\section{Acknowledgments}

The authors thank all participants who donated samples and the staff members who were involved in this research. We especially acknowledge the contributions of Young Ki Lee (Syntekabio, Inc., Republic of Korea) who worked on NGS data generation, and Eun Pyo Hong (Hallym University College of Medicine, Republic of Korea) who contributed to the evaluation of the functional importance of the gene mutations.

\section{Funding}

This research was supported by grant of the Korea Health Industry Development Institute (KHIDI) of the Ministry of Health \& Welfare (HI14C0072) and the National Research Foundation (NRF) of the Ministry of Education (2014R1A1A3053168), Republic of Korea.

\section{Availability of data and materials}

The data that support the findings are available in this published article and its supplementary information files; and the datasets analysed during the current study are available from the corresponding author on reasonable request.

\section{Authors' contributions}

PJW have made substantial contribution to design of the work, interpretation of data, writing the manuscript and revising it critically for important intellectual content. HSG and KJK participated in the analysis and interpretation of data and drafting the manuscript. KY and YS participated in the study design, data acquisition, and clinical data evaluation. KHL contributed to the design of the study. JJS oversaw generation of sequencing data and participated in writing of the manuscript. All co-authors approved the final version of the manuscript for publication.

\section{Competing interests}

The authors declare that they have no competing interests.

\section{Ethics approval and consent to participate}

The Institutional Review Board for Human Research at Seoul National University approved the study protocol (IRB number 1201-099-396), and all participants signed informed consent forms.

\section{Author details}

'Department of Medical Genetics, College of Medicine, Hallym University, 1 Hallymdaehak-gil, Chuncheon, Gangwon-do 24252, Republic of Korea. ${ }^{2}$ Wide River Institute of Immunology, Seoul National University, Hongcheon, Republic of Korea. ${ }^{3}$ Department of Internal Medicine, Seoul National University Hospital, Seoul, Republic of Korea. ${ }^{4}$ Omics Core Lab., National Cancer Center, Goyang, Republic of Korea. ${ }^{5}$ The Catholic University, Seoul, Republic of Korea. ${ }^{6}$ Syntekabio Inc., Seoul, Republic of Korea. ${ }^{7}$ Department of Biochemistry, School of Medicine, Ewha Womans University, Seoul, Republic of Korea.

Received: 24 January 2016 Accepted: 15 February 2017

Published online: 01 March 2017

\section{References}

1. Ferlay J, Soerjomataram I, Dikshit R, Eser S, Mathers C, Rebelo M, et al. Cancer incidence and mortality worldwide: sources, methods and major patterns in GLOBOCAN 2012. Int J Cancer. 2015;136:359-86.

2. Jung KW, Won YJ, Kong HJ, Oh CM, Seo HG, Lee JS. Cancer statistics in Korea: incidence, mortality, survival and prevalence in 2010. Cancer Res Treat. 2013:45:1-14.

3. Schlenk RF, Döhner K, Krauter J, Fröhling S, Corbacioglu A, Bullinger L, et al. Mutations and treatment outcome in cytogenetically normal acute myeloid leukemia. N Engl J Med. 2008;358:1909-18.

4. Patel JP, Gönen M, Figueroa ME, Fernandez $H$, Sun Z, Racevskis J, et al. Prognostic relevance of integrated genetic profiling in acute myeloid leukemia. N Engl J Med. 2012;366:1079-89.

5. Gregory TK, Wald D, Chen Y, Vermaat JM, Xiong Y, Tse W. Molecular prognostic markers for adult acute myeloid leukemia with normal cytogenetics. J Hematol Oncol. 2009;2:23.

6. Cancer Genome Atlas Research Network. Genomic and epigenomic landscapes of adult de novo acute myeloid leukemia. N Engl J Med. 2013;368:2059-74.

7. Ley TJ, Mardis ER, Ding L, Fulton B, McLellan MD, Chen K, et al. DNA sequencing of a cytogenetically normal acute myeloid leukaemia genome. Nature. 2008;456:66-72.

8. Mardis ER, Ding L, Dooling DJ, Larson DE, McLellan MD, Chen K, et al. Recurring mutations found by sequencing an acute myeloid leukemia genome. N Engl J Med. 2009;361:1058-66.

9. Ley $T J$, Ding L, Walter MJ, McLellan MD, Lamprecht $T$, Larson $\mathrm{DE}$, et al. DNMT3A mutations in acute myeloid leukemia. N Engl J Med. 2010:363:2424-33

10. Yan X-J, Xu J, Gu Z-H, Pan CM, Lu G, Shen Y, et al. Exome sequencing identifies somatic mutations of DNA methyltransferase gene DNMT3A in acute monocytic leukemia. Nat Genet. 2011;43:309-15.

11. Ding L, Ley TJ, Larson DE, Miller CA, Koboldt DC, Welch JS, et al. Clonal evolution in relapsed acute myeloid leukaemia revealed by whole-genome sequencing. Nature. 2012;481:506-10.

12. Patel RK, Jain M. NGS QC Toolkit: a toolkit for quality control of next generation sequencing data. PLoS One. 2012;7:e30619.

13. Li H, Durbin R. Fast and accurate long-read alignment with BurrowsWheeler transform. Bioinformatics. 2010;26:589-95.

14. Cibulskis K, Lawrence MS, Carter SL, Sivachenko A, Jaffe D, Sougnez C, et al. Sensitive detection of somatic point mutations in impure and heterogeneous cancer samples. Nat Biotechnol. 2013:31:213-9.

15. Koboldt DC, Zhang Q, Larson DE, Shen D, McLellan MD, Lin L, et al. VarScan 2: somatic mutation and copy number alteration discovery in cancer by exome sequencing. Genome Res. 2012;22:568-76.

16. Ramos AH, Lichtenstein L, Gupta M, Lawrence MS, Pugh TJ, Saksena G, et al. Oncotator: cancer variant annotation tool. Hum Mutat. 2015;36:E2423-2429.

17. Park S, Koh Y, Yoon SS. Effects of somatic mutations are associated with SNP in the progression of individual acute myeloid leukemia patient: the two-hit theory explains inherited predisposition to pathogenesis. Genomics Inform. 2013;11:34-7.

18. Lee $\mathrm{KH}, \mathrm{Lim} \mathrm{JH}, \mathrm{Kim} J \mathrm{H}$. Annotation of genes having candidate somatic mutations in acute myeloid leukemia with whole-exome sequencing using concept lattice analysis. Genomics Inform. 2013;11:38-45. 
19. Heo SG, Hong EP, Park JW. Genetic risk prediction for normal-karyotype acute myeloid leukemia using whole-exome sequencing. Genomics Inform. 2013;11:46-51.

20. Lawrence MS, Stojanov P, Polak P, Kryukov GV, Cibulskis K, Sivachenko A, et al. Mutational heterogeneity in cancer and the search for new cancerassociated genes. Nature. 2013;499:214-8.

21. Huang DW, Sherman BT, Lempicki RA. Systematic and integrative analysis of large gene lists using DAVID bioinformatics resources. Nature Protoc. 2009;4:44-57.

22. Kanehisa M, Goto S, Sato Y, Kawashima M, Furumichi M, Tanabe M. Data, information, knowledge and principle: back to metabolism in KEGG. Nucleic Acids Res. 2014;42:D199-205.

23. Dubrowinskaja N, Gebauer K, Peters I, Hennenlotter J, Abbas M, Scherer R, et al. Neurofilament Heavy polypeptide CpG island methylation associates with prognosis of renal cell carcinoma and prediction of antivascular endothelial growth factor therapy response. Cancer Med. 2014;3:300-9.

24. Kim MS, Chang X, LeBron C, Nagpal JK, Lee J, Huang Y, et al. Neurofilament heavy polypeptide regulates the Akt-beta-catenin pathway in human esophageal squamous cell carcinoma. PLoS One. 2010;5:e9003.

25. Wang Y, Martin TA, Jiang WG. HAVCR-1 expression in human colorectal cancer and its effects on colorectal cancer cells in vitro. Anticancer Res. 2013;33:207-14

26. Cuadros T, Trilla E, Sarró E, Vilà MR, Vilardell J, et al. HAVCR/KIM-1 activates the IL-6/STAT-3 pathway in clear cell renal cell carcinoma and determines tumor progression and patient outcome. Cancer Res. 2014;74:1416-28.

27. Li S, Xie Y, Zhang W, Gao J, Wang M, Zheng G, et al. Interferon alphainducible protein 27 promotes epithelial-mesenchymal transition and induces ovarian tumorigenicity and stemness. J Surg Res. 2015;193:255-64.

28. Rasmussen UB, Wolf C, Mattei MG, Chenard MP, Bellocq JP, Chambon P, Rio MC, Basset P. Identification of a new interferon-alpha-inducible gene (p27) on human chromosome $14 \mathrm{q} 32$ and its expression in breast carcinoma. Cancer Res. 1993:53:4096-101.

29. Demidyuk IV, Shubin AV, Gasanov EV, Kurinov AM, Demkin W, Vinogradova $T V$, et al. Alterations in gene expression of proprotein convertases in human lung cancer have a limited number of scenarios. PLoS One. 2013;8:e55752.

30. Trojani A, Di Camillo B, Tedeschi A, Lodola M, Montesano S, Ricci F, et a. Gene expression profiling identifies ARSD as a new marker of disease progression and the sphingolipid metabolism as a potential nove metabolism in chronic lymphocytic leukemia. Cancer Biomark. 2011-2012:11:15-28.

31. Lu GD, Ang YH, Zhou J, Tamilarasi J, Yan B, Lim YC, et al. CCAAT/enhancer binding protein a predicts poorer prognosis and prevents energy starvation-induced cell death in hepatocellular carcinoma. Hepatology. 2015;61:965-78.

32. Fasan A, Haferlach C, Alpermann T, Jeromin S, Grossmann V, Eder C, et al. The role of different genetic subtypes of CEBPA mutated AML. Leukemia. 2014:28:794-803.

33. Macher-Goeppinger S, Bermejo JL, Schirmacher P, Pahernik S, Hohenfellne M, Roth W. Senescence-associated protein p400 is a prognostic marker in renal cell carcinoma. Oncol Rep. 2013;30:2245-53.

34. Mattera L, Escaffit F, Pillaire MJ, Selves J, Tyteca S, Hoffmann JS, et al. The p400/Tip60 ratio is critical for colorectal cancer cell proliferation through DNA damage response pathways. Oncogene. 2009;28:1506-17.

35. Sacco JJ, Yau TY, Darling S, Patel V, Liu H, Urbé S, et al. The deubiquitylase Ataxin-3 restricts PTEN transcription in lung cancer cells. Oncogene. 2014;33:4265-72

36. Revill K, Wang T, Lachenmayer A, Kojima K, Harrington A, Li J, Powers S, et al. Genome-wide methylation analysis and epigenetic unmasking identify tumor suppressor genes in hepatocellular carcinoma. Gastroenterology. 2013;145:1424-35.e1-25.

37. Szebenyi G, Smith GM, Li P, Brady ST. Overexpression of neurofilament H disrupts normal cell structure and function. J Neurosci Res. 2002;68:185-98.

38. Okumura Y, Takahashi E, Yano M, Ohuchi M, Daidoji T, Nakaya T, et al. Novel type II transmembrane serine proteases, MSPL and TMPRSS13, Proteolytically activate membrane fusion activity of the hemagglutinin of highly pathogenic avian influenza viruses and induce their multicycle replication. J Virol. 2010;84:5089-96.

39. Hashimoto T, Kato M, Shimomura T, Kitamura N. TMPRSS13, a type transmembrane serine protease, is inhibited by hepatocyte growth factor activator inhibitor type 1 and activates pro-hepatocyte growth factor. FEBS J. 2010;277:4888-900.
40. Lin F, Zhang PL, Yang XJ, Shi J, Blasick T, Han WK, et al. Human kidney injury molecule-1 (hKIM-1): a useful immunohistochemical marker for diagnosing renal cell carcinoma and ovarian clear cell carcinoma. Am J Surg Pathol. 2007;31:371-81.

41. Hsiao C-P, Araneta M, Wang XM, Saligan LN. The association of IFI27 expression and fatigue intensification during localized radiation therapy: implication of a para-inflammatory bystander response. Int J Mol Sci. 2013;14:16943-57.

42. Naing A, Reuben JM, Camacho LH, Gao H, Lee B-N, Cohen EN, et al. Phase I Dose Escalation Study of Sodium Stibogluconate (SSG), a Protein Tyrosine Phosphatase Inhibitor, Combined with Interferon Alpha for Patients with Solid Tumors. J Cancer. 2011:2:81-9.

43. Sun X, Essalmani R, Seidah NG, Prat A. The proprotein convertase PC5/6 is protective against intestinal tumorigenesis: in vivo mouse model. Mol Cancer. 2009;8:73

44. McElhinny AS, Li J-L, Wu L. Mastermind-like transcriptional co-activators: emerging roles in regulating cross talk among multiple signaling pathways. Oncogene. 2008:27:5138-47.

45. Heynen GJJE, Nevedomskaya E, Palit S, Jagalur Basheer N, Lieftink C, Schlicker A, et al. Mastermind-Like 3 Controls Proliferation and Differentiation in Neuroblastoma. Mol Cancer Res MCR. 2016;14:411-22.

46. Wagner EF, Nebreda AR. Signal integration by JNK and p38 MAPK pathways in cancer development. Nat Rev Cancer. 2009:9:537-49.

\section{Submit your next manuscript to BioMed Central and we will help you at every step:}

- We accept pre-submission inquiries

- Our selector tool helps you to find the most relevant journal

- We provide round the clock customer support

- Convenient online submission

- Thorough peer review

- Inclusion in PubMed and all major indexing services

- Maximum visibility for your research

Submit your manuscript at www.biomedcentral.com/submit
) Biomed Central 